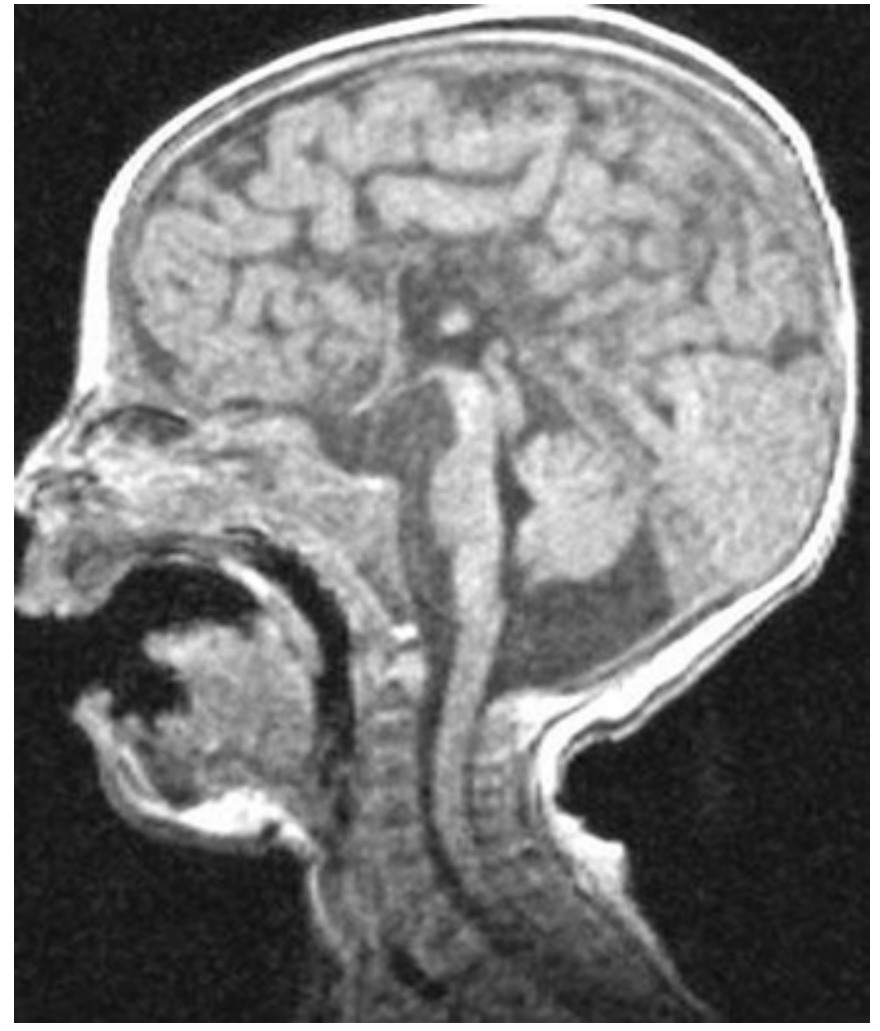

Abstract 545 Figure 2

\section{A GIANT ORBITAL PLEXIFORM NEUROFIBROMA WITH MASSIVE INTRACRANIAL EXTENTION IN A NEWBORN}

doi:10.1136/archdischild-2012-302724.0546

${ }^{1} \mathrm{~N}$ Yazici, ${ }^{2} \mathrm{H}$ Gulcan, ${ }^{3} \mathrm{O}$ Alkan, ${ }^{4} \mathrm{R}$ Yaycioglu, ${ }^{5} \mathrm{~N}$ Bal, ${ }^{1} \mathrm{~F}$ Sarialioglu. ${ }^{1}$ Department of Pediatric Oncology; ${ }^{2}$ Department of Neonatology; ${ }^{3}$ Department of Radiology; ${ }^{4}$ Department of Eye Disorders; ${ }^{5}$ Department of Pathology, Baskent University, Adana, Turkey

Orbital masses in newborns are rare. Herein a newborn with a giant orbital tumor is presented. A 10-day female baby was admitted to hospital with proptosis. She was the first child of a 29 year-old mother, was born from an uneventful pregnancy. Her birth weight was 3000 gr. No consanguinity between the parents and history of neurofibromatosis in family were present. In physical examination, the baby had bilateral prominent proptosis and rest of the physical examination was unremarkable. The initial diagnosis was metastatic neuroblastoma. MRI of the brain showed a huge mass involving bilateral cavernous sinus, perimedullary cistern, orbita and orbital apex. Neuroblastoma markers including urine VMA, NSE, bone-marrow aspiration examination, abdominal ultrasonography and a two-diamentional chest x-ray were normal. Although the initial radiological diagnosis was plexiform neurofibroma, an open biopsy was performed to rule out other possibilities specially orbital malignant tumors. Histopathological diagnosis was a typical plexiform neurofibroma. The child has been treated with palliative measures and for seizures. Although it was a benign histology, the clinical picture was drastic Like an infant with a malignant tumor chemotherapy was planned. But the family refused chemotherapy and any other form of antineoplastic therapy. In conclusion, the plexiform neurofibroma must be taken in consideration in newborn infant with orbital tumors.
${ }^{1} \mathrm{SI}$ lurian, ${ }^{2} \mathrm{~S}$ Iurian, ${ }^{3} \mathrm{G}$ Gradinariu, ${ }^{3} \mathrm{~A}$ Vidrighin, ${ }^{3} \mathrm{~B}$ Mehedintu. ${ }^{1}$ Research Department, Pediatric Clinic, Lucian Blaga University; ${ }^{2}$ Clinical Laboratory, Pediatric Hospital; ${ }^{3}$ Pediatric Clinic, Sibiu, Romania

Background and Aims Guillain-Barré syndrome (GBS) is an acute polyradiculoneuropathy with weakness and diminished reflexes. The authors present clinical peculiarities in 2 cases diagnosed with GBS

Methods The authors present 2 cases: a 18 month-old male admitted because of unstable walking ( $1^{\text {st }}$ case) and severe lower extremities pain for the $2^{\text {nd }}$ case ( 5 year-old boy).

Cases history: upper respiratory tract illness 3 weeks before symptoms onset; no vaccinations, surgical procedures or trauma prior to disease.

\section{Clinical exam:}

1. $1^{\text {st }}$ case presented respiratory signs (dysphonia, slurred speech, short breath);

2. $2^{\text {nd }}$ case was admitted for severe legs pain.

In addition, both cases were characterized by symmetrical extremities weakness, legs sensory changes (paresthesias, numbness), intense nuchal rigidity, positive Brudzinski sign, abolished osteo-tendinous reflexes in both upper/lower limbs and no abdominal reflexes. There were performed electromyography (EMG), nerve conduction velocity tests (NCT), serologic and cerebrospinal fluid (CSF) analysis.

Results CSF analysis identified albumino-cytologic dissociation: elevation of CSF protein with normal white blood cells count. The serologic studies shown normal titers for cytomegalovirus, EpsteinBarr virus and Mycoplasma. The NCT and EMG have proved severe demyelinating neuropathy and distal conduction block. Differential diagnosis: authors excluded meningitis, myopathies, poliomyelitis, polymyositis and myasthenic syndromes. The patients were treated with intravenous immunoglobulins with good clinical evolution.

Conclusions The authors presented 2 cases with GBS secondary to respiratory infections. Cases peculiarities.

1. Even though small children have the lowest risk, $1^{\text {st }}$ case presented very early onset;

2. Both cases presented intense nuchal rigidity.

\section{LINEAR GROWTH AND BODY MASS INDEX IN INFANTS AND CHILDREN AFTER CAUSTIC INGESTION}

doi:10.1136/archdischild-2012-302724.0548

'A Soliman, ${ }^{2} \mathrm{~W}$ Saleem, ${ }^{1} \mathrm{~A}$ Elawwa, ${ }^{1} \mathrm{M}$ Wagdy, ${ }^{1} \mathrm{H}$ Abdelrahman. ${ }^{1}$ Pediatrics; ${ }^{2}$ Pediatric Emergency, Hamad Medical Center, Doha, Oatar

Caustic injuries continue to be a significant morbidity in the pediatric patient group. Management of caustic ingestion in children remains a difficult challenge, with the outcome ranging from an asymptomatic state to esophageal strictures and variable effects on linear growth and weight gain.

We recorded and analyzed the growth data of 10 children ranging in age from 1 to 4 years with caustic ingestion presented from 2005 to 2007 and treated at Hamad Medical Center. Initial management consisted of prompt endoscopy and early institution of steroids and antibiotics. The decision on esophagoscopy was made on the basis of drooling and dysphagia. Significant esophageal burns were confirmed in all of them and subsequently five of them were managed successfully by repeated dilation due to multiple strictures.

None of the patients had underweight and/or stunting for 2 years after treatment. However, the BMI decreased from 16.77 +/$3.5 \mathrm{~kg} / \mathrm{m} 2$ to $16.26+/-2.9 \mathrm{~kg} / \mathrm{m} 2$ and the height standard deviation score (HtSDS) decreased significantly from (-) $0.09+/-0.99$ to (-) $0.58+/-1$. Children with multiple strictures that required 
repeated dilatation had significantly decreased HtSDS after 2 years of ingestion versus those with no significant strictures and vs controls.

In conclusion, proper management of strictures and nutritional support appears to decrease subsequent growth problems, those with multiple strictures had slower linear growth.

\section{PREVENTION OF OBESITY THROUGH HEALTHY NUTRITION IN THE FIRST YEAR OF LIFE}

doi:10.1136/archdischild-2012-302724.0549

ML Neamtu, L Dobrota, BI Mehedintu. Pediatric Clinic Hospital Sibiu, Lucian Blaga University of Sibiu, Sibiu, Romania

Background Obesity, defined as excess body fat can be diagnosed at 6 months. In children, BMI percentiles are used, defining the overweight between 85-95 and obesity $>95$. The main predictors of primary obesity in infancy are high birthweight, parental obesity, low total energy expenditure and energy intake (EI).

Aim Calculation of optimal EI in infants.

Method Calculation of estimated energy requirements (EER) for infants was made according to the formulas: [(89xGkg)-100]+175 between 0-3 months, [(89xGkg)-100]+56, between 4-6 months, $[(89 \times \mathrm{Gkg})-100]+22$, between $7-12$ months. Calculation of fluid requirements was made according to Apert formula: G/10+200(300). Results Were studied 11 infants 7-9 months old with normal birthweight, without parental obesity, fed with formula $(76 \mathrm{kcal} / 100 \mathrm{ml})$ from the first month, with diversification started from 4 months, eating high-caloric foods from 5 months $(100 \mathrm{kcal} / 100 \mathrm{ml})$, fed according to Apert formula. Seven infants had weight above the $85^{\text {th }}$ percentile and 4 of them $>95^{\text {th }}$ percentile. Comparing EI according to Apert formula with EER is found an excess, increased with the beginning of diversification. Disposal of high-caloric foods, feeding with formulas with a similar amount of calories as human milk (67kcal $/ 100 \mathrm{ml})$, feeding according to EER and fluid supplementation with low-caloric beverages have led to normalization of BMI percentiles of infants at the age of 12 months

Conclusions EI is an important predictor of infant weight. It is necessary to use formulas with a similar amount of calories as human milk. Infant feeding according to EER and fluid supplementation with low-caloric beverages lead to a normal BMI.

\section{GROWTH ASSESSMENT IN CHILDREN AFTER ACUTE SEPTIC VERSUS ASEPTIC MENENGITIS: A CONTROLLED STUDY}

doi:10.1136/archdischild-2012-302724.0550

A Soliman, W Seleem, M Wagdy, A Elawwa. Pediatrics, Hamad Medical Center, Doha, Oatar

We recorded and analyzed the growth data of 40 children with acute meningitis (age $5.8+/-3.1$ years) for a year or more after treatment and compared them with their age and sex matched healthy siblings $(n=100)$. None of the patients had meningitis sequelae that could affect linear growth. None of them had underweight and/or stunting for one year or more after treatment. No difference in dietary intake between patients and controls. The height standard deviation scores (HtSDS) of patients decreased significantly from $-0.06+/-0.95$ at the onset of meningitis to $-0.46+/-1$ after $>1$ year of follow-up and were significantly lower than those for their normal siblings $(0.31+/-0.5)$. Fifteen out of the 40 patients had decreased HtSDS $>-0.5$, while 3 had decreased HtSDS $>-1$ after $>1$ year of follow-up. The BMI of patients significantly increased after 1 year or more of the acute attack but did not differ from the BMI for the controls. One patient and none of the controls had BMISDS $>2$ at presentation. $5 / 40$ patients and $2 / 100$ children from the control group had BMISDS > 2 after 1 year or more of follow-up. The HtSDS decreased and BMI increased significantly in both groups with septic $(n=10)$ and aseptic meningitis $(n=30)$ with no significant difference among the 2 groups.

In conclusion, long term growth delay and overweight appear to be risk factors following an acute attack of both septic and aseptic meningitis.

\section{THE IMPORTANCE OF PROPER NUTRITION CHILDREN: FROM CONCEPTION TO PUBERTY}

doi:10.1136/archdischild-2012-302724.0551

\section{B Stanimirov. Pediatrics, Health Centre Novi Sad, Novi Sad, Serbia}

Background The proper and healthy diet has become one of the most important problems of modern man, and for proper growth and development of children's body, it is much earlier, before and during pregnancy, a healthy respect life. Nutrition styles in pregnancy should be the quality and variety that is. to bring all the nutrients in the medium quantity. Role the importance of proper nutrition especially true in childhood, when it comes to the most intense growth and development, and health is the foundation for a lifetime. Children and adolescents go through different periods of intense growth and development: the infant period, the period of early childhood (up to 3 years), preschool age (3 to 6 years), school age (6 to 13 years) and adolescence to 19 years. Quality of food is reflected in the following and applying the principles of proper nutrition: rationality, optimality and diversity means that nutrition during the day should provide enough energy for growth, development and physical activity of children. Energy requirements vary by age, are equal to 9 years of age, and in adolescence are different in relation to sex. The most common are: cereals and cereal products (30\%), milk and milk products $(25 \%)$, meat, fish and eggs $(20 \%)$, vegetables, fruits, fats and oils (10\%) and sugar and concentrated sweets (5\%).

Conclusion Proper nutrition is a habit among the most important things a child learns, with long-term effect on the health and welfare, which is good for the prevention of obesity, malnutrition and chronic diseases.

\section{A BABY IN A PICKLE}

doi:10.1136/archdischild-2012-302724.0552

\section{J Clegg, M Thorpe. Child Health Dept, Royal Cornwall Hospital, Truro, UK}

Background and Aims Abdominal distention and feeding difficulties are common in the preterm. Many infants receive treatment for non-proven necrotising enterocolitis (NEC) due to the devastating consequences if missed. Positive alternative diagnoses for abdominal distention are relevant.

Methods We report a case of acute abdominal distension in a 29 week gestation, $750 \mathrm{~g}$ infant receiving expressed breast milk from a mother who had a craving for pickled onions, and discuss the relevant literature.

Results The infant had mild respiratory distress syndrome at birth. He reached full enteral feed volumes by day 10 . On day 20 he developed distension of the abdomen and apparent abdominal pain. 